\title{
Ein neuer Weg zur Bewertung der Recyclingfähigkeit von Produkten mittels Statistischer Entropie: Fallbeispiel Mobiltelefone
}

\author{
Caroline Roithner · Oliver Cencic · Helmut Rechberger
}

Angenommen: 17. Juni 2021 / Online publiziert: 9. Juli 2021

(C) Der/die Autor(en) 2021

Zusammenfassung Statistische Entropie kann zur Bewertung von unterschiedlichen Verfahren der Abfallwirtschaft herangezogen werden. Führt das Verfahren $\mathrm{zu}$ einer Separierung und damit Konzentrierung einzelner Materialien oder Stoffe, so entspricht dies einer niedrigen Statistischen Entropie, kommt es jedoch zu einer Vermischung und Verdünnung, erhöht sich diese. Recyclingverfahren haben beispielsweise das Ziel, konzentrierte Recyclingoutputs aus einem gemischten Abfallinput zu generieren, was einer Reduzierung der Statistischen Entropie entspricht. Eine Bewertung mittels Statistischer Entropie kann jedoch auch für Produkte selbst verwendet werden. Hierbei werden die Materialzusammensetzung und der Aufbau von Produkten mittels Statistischer Entropie bewertet. Komplexe Produkte, die aus einer Vielzahl von Materialien bestehen, weisen tendenziell eine höhere Statistische Entropie und eine schlechtere Recyclingfähigkeit auf als Produkte, die nur aus einigen wenigen Materialien zusammengesetzt sind. Die Statistische Entropie kann daher als Maß für die Recyclingfähigkeit von Produkten betrachtet werden. Anhand einer Fallstudie zu unterschiedlichen Generationen von Mobiltelefonen wird die Anwendung der neuen Bewertungsmethode demonstriert. Die Ergebnisse der Fallstudie zeigen, dass sich die Bewertung mittels Statistischer Entropie dafür eignet, die produktinhärente Recyclingfähigkeit von Produkten $\mathrm{zu}$ bewerten und dabei wichtige Einblicke in das Design for Recycling von Produkten zu liefern. Als Planungs- bzw.

\section{Roithner, MSc $(\bowtie)$}

DI Dr. O. Cencic

Univ.-Prof. DI Dr. H. Rechberger Institut für Wassergüte und Ressourcenmanagement, Technische Universität Wien, Karlsplatz 13/226, 1040 Wien, Österreich caroline.roithner@tuwien.ac.at
Entscheidungstool angewandt, könnte der neue Bewertungsansatz helfen, die Weichen für eine effektive Kreislaufwirtschaft bereits im Produktdesign zu stellen und so in Folge ein besseres Recycling zu ermöglichen.

Schlüsselwörter Recyclingfähigkeit · Statistische Entropie · Produktdesign Mobiltelefone $\cdot$ Kreislaufwirtschaft

A new way of assessing the recyclability of products using statistical entropy: Case study mobile phones

Abstract Statistical entropy can be used to evaluate different waste management processes. If a process leads to separation and thus concentration of individual materials or substances, this corresponds to low statistical entropy, which increases if mixing and dilution occur. For example, recycling processes aim to generate concentrated recycling outputs from a mixed waste input, which corresponds to a reduction in statistical entropy. However, a statistical entropy assessment can also be used for products themselves, where the material composition and the product structure are evaluated. Complex products that consist of many materials tend to have a higher statistical entropy and are less recyclable than products composed of only a few materials. Statistical entropy can therefore be viewed as a measure of the recyclability of products. The application of the new assessment method is demonstrated in a case study on mobile phones of different generations. The results of the case study show that statistical entropy is suitable for evaluating the productinherent recyclability of products and thus providing important insights into the design for recycling of products. Applied as a planning or decision-making tool, the new assessment approach could help to set the course for an ef- fective Circular Economy as early as in the product design and thus enable better recycling.

Keywords Recyclability - Statistical Entropy · Product design · Mobile phones · Circular Economy

\section{Einleitung}

Die Europäische Union (EU) forciert die Etablierung einer Kreislaufwirtschaft (im weiteren $\mathrm{CE}$ für Circular Economy), in der Produkte bzw. Materialien bestmöglich im Kreislauf gehalten werden (European Commission 2015a, b; European Union 2020). Dabei wird erstmals auch dem Produktdesign eine große Bedeutung zugeschrieben. So ist feststellbar, dass der Aufbau und die Zusammensetzung von Produkten einen wesentlichen Einfluss auf das Recycling bzw. die Kreislaufführung haben. Komplex aufgebaute Produkte, wie beispielsweise Elektrogeräte, verursachen folglich einen höheren, oft nicht wirtschaftlichen Recyclingaufwand, als Produkte, die einfacher gestaltet sind.

Insbesondere in der Produktgruppe der Elektrogeräte haben Vielfalt und Komplexität der Geräte durch die rasante technologische Entwicklung stark zugenommen (Singh et al. 2018). Die damit verbundene Miniaturisierung von Bauteilen führt oft $\mathrm{zu}$ einer schlechteren Zerlegbarkeit und so $\mathrm{zu}$ Verlusten von wertvollen Rohstoffen, deren Rückgewinnung nicht möglich ist oder sich nicht lohnt. Der Druck auf die Recyclingindustrie wächst stetig, da immer neue Herausforderungen auftreten. Es scheint daher notwendig, die Auswirkungen des Produktdesigns auf die Recyclingfähigkeit von Produkten näher zu betrachten.

Roithner und Kollegen haben eine Bewertungsmethode entwickelt, die basierend auf Produktinformationen wie Materialzusammensetzung und Aufbau, eine produktinhärente Recy- 


\section{Originalarbeit}

Smartphone (117.65 g)

Leiterplatte mit elektronischen Bauteilen

Leiterplatte | Kondensatoren | Halbleiter | Tasten | Fassungen | Lot

Gehäuse

Bildschirm

Batterie

Kathode | Anode | Separator | Elektrolyt | Hülle

Kameras

Linsen | Diverses (Elektronik, Drähte, etc.) | Lot | Hülle

SIM-Kartenträger

Abdeckung

Rückseitenabdeckung | Kleber

Tasten

Tasten | Halbleiter | Diverses (Elektronik, Drähte, etc.) | Lot

Schrauben

Schrauben selbst | Kleber

Lautsprecher

Magnete | Diverses (Elektronik, Drähte, etc.) | Lot | Hülle

Vibrationsmotor

Magnete | Diverses (Elektronik, Drähte, etc.) | Kleber | Lot | Hülle

Abb. 1 Produktaufbau der modellierten Mobiltelefone

\section{Mobiltelefon (93.10 g)}

Leiterplatte mit elektronischen Bauteilen

Leiterplatte | Kondensatoren | Halbleiter | Fassungen | Lot Lautsprecher:

Magnete | Diverses | Lot | Hülle

Vibrationsmotor:

Magnete | Diverses | Lot | Hülle

Gehäuse

Gehäuse selbst | Rahmen um Gehäuse

Bildschirm

Batterie

Kathode |Anode | Elektrolyt | Hülle

Kamera

Linse | Diverses (Elektronik, Drähte, etc.) | Lot | Hülle

SIM-Kartenträger

Abdeckungen

Rückseitenabdeckung | Batterieabdeckung

Tasten

Tasten | Diverses (Elektronik, Drähte, etc.) | Lot

Schrauben

Tastatur

Tastatur selbst | Tastaturmodul 
clingfähigkeit errechnet (Roithner et al. 2021, Publikation eingereicht). Im Gegensatz zu anderen Methoden beziehen sich Roithner et al. (2021) rein auf Informationen des Produktdesigns. Ziel ist es, eine Bewertungsgrundlage für ProduktdesignerInnen und ProduzentInnen zu schaffen, die es ermöglicht, Produkte recyclingfähiger $\mathrm{zu}$ gestalten bzw. Produkte zu vergleichen. In dieser Arbeit wird der Bewertungsansatz von Roithner et al. (2021) auf zwei Mobiltelefone aus unterschiedlichen technologischen Produktgenerationen angewendet und gezeigt, dass dadurch Unterschiede in der produktinhärenten Recyclingfähigkeit festgestellt sowie Verbesserungspotenziale abgeleitet werden können.

\section{Material und Methoden}

\subsection{Modellierung von Mobiltelefonen}

Da Originaldaten zu Mobiltelefonen nur sehr schwer zugänglich sind, wurde im Vorfeld der Modellierung eine Literaturrecherche durchgeführt, anhand deren Ergebnissen zwei realitätsnahe Mobiltelefone zusammengestellt wurden (Tarantili et al. 2010; Palmieri et al. 2014; Ueberschaar et al. 2017; Tan et al. 2017; Bookhagen et al. 2018, 2020; Holgersson et al. 2018; Singh et al. 2018; Smodiš et al. 2018; Fontana et al. 2019; Liu et al. 2019). Die modellierten Mobiltelefone umfassen einerseits ein Mobiltelefon, das mit einem Erscheinungsjahr um 2008 datiert werden kann, und andererseits ein Smartphone (dieses stellt modernere Mobiltelefone mit einem Touchscreen dar) mit einem

Tab. 1 Massen und Anzahl der Materialien der modellierten Mobiltelefone und deren Komponenten

\begin{tabular}{|c|c|c|c|c|c|}
\hline & Masse $(\mathrm{g})$ & $\begin{array}{l}\text { Material- } \\
\text { anzahl }\end{array}$ & & Masse $(\mathrm{g})$ & $\begin{array}{l}\text { Material- } \\
\text { anzahl }\end{array}$ \\
\hline $\begin{array}{l}\text { Leiterplatte mit elektr. } \\
\text { Bauteilen }\end{array}$ & 12,15 & 42 & $\begin{array}{l}\text { Leiterplatte mit elektr. } \\
\text { Bauteilen }\end{array}$ & 15,79 & 19 \\
\hline Gehäuse & 20,00 & 1 & Gehäuse & 9,00 & 1 \\
\hline Bildschirm & 32,00 & 3 & Bildschirm & 7,53 & 2 \\
\hline Batterie & 26,00 & 10 & Batterie & 19,01 & 7 \\
\hline Kameras & 5,00 & 17 & Kamera & 9,00 & 7 \\
\hline SIM-Kartenträger & 2,00 & 1 & SIM-Kartenträger & 3,00 & 1 \\
\hline Abdeckung & 13,00 & 2 & Abdeckungen & 15,00 & 1 \\
\hline Tasten & 2,00 & 16 & Tasten & 3,00 & 7 \\
\hline Schrauben & 2,00 & 2 & Schrauben & 3,00 & 1 \\
\hline Lautsprecher & 1,50 & 13 & - & - & - \\
\hline Vibrationsmotor & 2,00 & 15 & - & - & - \\
\hline- & - & - & Tastatur & 8,76 & 7 \\
\hline Smartphone & 117,65 & 49 & Mobiltelefon & 93,10 & 24 \\
\hline
\end{tabular}

Diverses, Lot und Hülle), Fassungen und Lot. Im Gegensatz zum Smartphone sind beim Mobiltelefon der Lautsprecher und der Vibrationsmotor an der Leiterplatte angebracht, jedoch einzeln abtrennbar. Weiters unterscheiden sich die Mobiltelefone in Bezug auf die Tastatur; beim Mobiltelefon stellt diese eine eigene Komponente dar, während beim Smartphone die Funktion der Tastatur vom Bildschirm (Touchscreen) sowie Bauteilen der Leiterplatte übernommen wird. Das Smartphone hat eine größere Masse als das Mobiltelefon $(117,65 \mathrm{~g}>93,10 \mathrm{~g})$. Die Massen der einzelnen Komponenten der Mobiltelefone und deren Materialzusammensetzung (Nm ... Anzahl der Materialien) sind in Tab. 1 gelistet. Es wurden nur jene Materialien betrachtet, die für die Analyse massemäßig relevant sind und in Summe mehr als $99 \%$ der Produktmasse ausmachen. Dadurch setzt sich das Smartphone aus 49 Materialien, das Mobiltelefon aus 24 Materialien zusammen. Der Begriff Materialien deckt dabei sowohl chemische Elemente (z. B. Al), Verbindungen (z.B. PVC) als auch Werkstoffe (z.B. Glas) ab. Die deutlichen Unterschiede im Produktaufbau sowie der Materialzusammensetzung können u.a. auf die zeitliche Weiterentwicklung von Mobiltelefonen zurückgeführt werden (Bsp. Touchscreen vs. Tastatur). Es ist an dieser Stelle festzuhalten, dass für die Materialliste der modellierten Mobiltelefone kein Anspruch auf Vollständigkeit erhoben wird.

\subsection{Statistische Entropie Analyse}

Die Statistische Entropie (SE), welche ihren Ursprung in der Thermodynamik hat, wurde in den letzten Jahrzehnten in unterschiedlichen Fachbereichen weiterentwickelt und angewandt. SE ist allgemein gesprochen ein $\mathrm{Ma}$ für die Ordnung bzw. Unordnung eines Systems. Je größer die Unordnung des betrachteten Systems, desto höher ist die SE. In den meisten Anwendungen ist es das Ziel, die SE gering zu halten und damit eine höhere Unordnung zu vermeiden. Einen wesentlichen Beitrag zur Weiterentwicklung von SE lieferte Claude Shannon, indem er SE in die Informationstheorie einführte und als den mittleren Informationsgehalt einer Nachricht definierte (Shannon 1948). Dabei ist der Informationsgehalt eines Zeichens (ausgedrückt in der Einheit Bit) umso geringer, je häufiger es in ei- 
ner Nachricht vorkommt (ausgedrückt durch die Auftretenswahrscheinlichkeit $\mathrm{p}_{\mathrm{i}}$ ). Shannons Ansatz kann vereinfacht mit Gl. 1 dargestellt werden (ld ist der Logarithmus zur Basis 2; es gilt $\operatorname{ld}(0)=0)$.

$$
H=-\sum p_{i} \operatorname{ld}\left(p_{i}\right)
$$

Shannons Ansatz wurde von Rechberger und Brunner weiterentwickelt, um konzentrierende bzw. verdünnende Effekte von Prozessen auf bestimmte Stoffe zu untersuchen und damit Materialflusssysteme bewerten zu können (Rechberger 1999; Rechberger und Brunner 2002). Dadurch können z.B. bei einer Müllverbrennungsanlage (mit einem Input und den Outputflüssen Schlacke, Schrott, Filterasche, Filterkuchen, Gips, Abwasser und Abgas) die Auswirkungen des Verbrennungsprozesses auf einen bestimmten Schadstoff im Abfallinput beurteilt werden. Liegt der Schadstoff nach Durchlaufen der Müllverbrennungsanlage in allen Outputflüssen in etwa der gleichen Konzentration vor, wäre dies der schlechteste Fall $(\mathrm{H}=$ Maximum). Kommt es jedoch zur maximalen Konzentrierung des Schadstoffes in einem einzigen Outputfluss, entspricht dies dem besten Fall $(\mathrm{H}=$ Minimum). In der Praxis ist die Stoffverteilung zwischen diesen beiden SE-Extremen zu finden.

Nachfolgende SE Anwendungen befassten sich u.a. mit der Bewertung von Stoffhaushaltssystemen (Rechberger und Graedel 2002; Sobańtka et al. 2014; Laner et al. 2017; Tanzer und Rechberger 2020) sowie Recyclingprozessen und Systemen im Sinne der CE (Parchomenko et al. 2020; Roithner und Rechberger 2020). Zuletzt wurde in der Publikation von Roithner et al. (2021) ein SE-Ansatz zur Bewertung von Produkten und deren produktinhärente Recyclingfähigkeit beschrieben.

\subsection{Produktinhärente Recyclingfähigkeit}

Für den nachfolgenden Mobiltelefonvergleich wurde der SE-basierte Bewertungsansatz von Roithner et al. (2021) herangezogen. Bei diesem Ansatz wird anhand der Materialverteilung und des Aufbaus von Produkten eine relative produktinhärente Recyclingfähigkeit (RPR) bestimmt. Es wird davon ausgegangen, dass diese Produktinformationen, die bereits in der Phase des Produktdesigns festgelegt werden, die

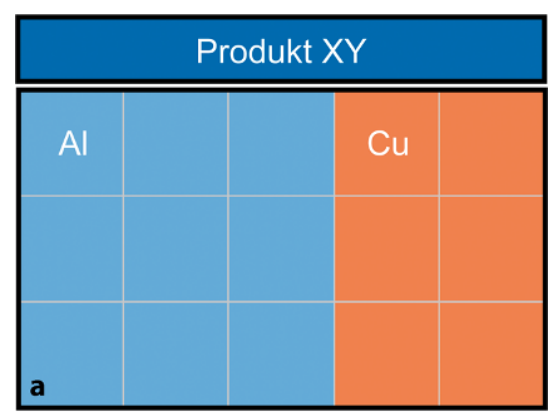

Information zum Produktaufbau

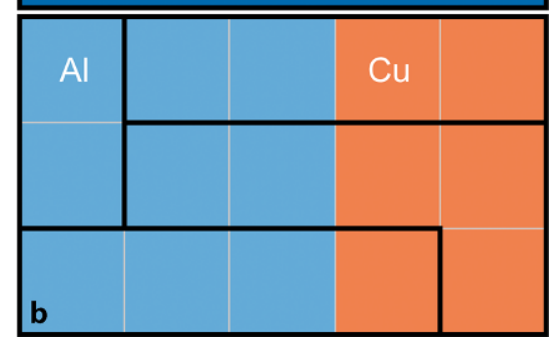

Abb. 2 Materialverteilung eines theoretischen Produkts XY ohne (oben) und mit (unten) Berücksichtigung des Produktaufbaus

Recyclingfähigkeit eines Produkts wesentlich prägen. Die Recyclingfähigkeit sinkt, je mehr Materialien in ähnlichen Konzentrationen im Produkt bzw. seinen Bauteilen vorliegen. Je stärker ein Produkt zerlegt werden kann, desto höher ist die Wahrscheinlichkeit, dass die Anzahl der Materialien in diesen Bauteilen abnimmt und manche dieser Materialien in höherer Konzentration vorliegen, wodurch die Recyclingfähigkeit steigt.

Da vorangegangene SE-Anwendungen bereits demonstrierten, dass SE eine geeignete Maßzahl für die Bewertung von Materialverteilungen ist (vgl. voriges Kapitel), wurde Shannons SE-Gleichung (vgl. Gl. 1) von Roithner et al. (2021) weiterentwickelt und für die oben erwähnten Produktinformationen ausgelegt. Anhand von Abb. 2 wird das Prinzip dieser Weiterentwicklung vereinfacht dargestellt.

Ein theoretisches Produkt XY besteht demnach zu neun Teilen aus Aluminium (Al) und $\mathrm{zu}$ sechs Teilen aus Kupfer (Cu) (vgl. Abb. 2). Im Falle, dass das Produkt nicht zerlegt werden kann, wäre die SE für diese Produktbewertung relativ hoch, da $\mathrm{Al}$ und $\mathrm{Cu}$ in ähnlich hoher Konzentration vorkommen. Ist eine Zerlegung in einzelne Produktelemente möglich, kann das Produkt jedoch in die vier dargestellten
Produktelemente zerlegt werden (vgl. schwarze Einrahmungen in der unteren Grafik von Abb. 2). Dies führt zu einer genaueren Information über die Verteilung von $\mathrm{Al}$ und $\mathrm{Cu}$ im Produkt bzw. den Produktelementen, wodurch eine bessere Abschätzung der produktinhärenten Recyclingfähigkeit ermöglicht wird. Beispielsweise besteht ein Produktelement aus reinem Aluminium (Konzentration $\mathrm{c}=1 \mathrm{~g} \mathrm{Al} / \mathrm{g}$ Produktelement), was den bestmöglichsten Fall für die Recyclingfähigkeit darstellt, da dieses Reinmaterial nach Ausbau direkt verwertet werden kann.

In Anlehnung an Shannons Ansatz haben Roithner et al. (2021) die Berechnung der sogenannten Relativen produktinhärenten Recyclingfähigkeit (RPR) gemäß Gl. 2 und 3 definiert (weitere Erklärung siehe Roithner et al. 2021). Wichtig ist dabei, dass für die Berechnung der produktelementspezifischen $\mathrm{SE}\left(\mathrm{H}_{\mathrm{j}}\right)$ (vgl. Gl. 2) jene $\mathrm{N}_{\mathrm{e}}$ Produktelemente berücksichtigt werden, die sich bei einer bestmöglichen Zerlegung ergeben. Die SE des gesamten Produkts $\left(\mathrm{H}_{\mathrm{p}}\right.$; vgl. Gl. 3$)$ ist das massengewichtete Mittel aller $H_{j}\left(M_{j}\right.$ ... Masse des Produktelements; $\mathrm{M}_{\mathrm{p}}$ ... Masse des gesamten Produkts). Für das finale RPR-Ergebnis (vgl. Gl. 4) wird die relative SE herangezogen, welche $\mathrm{H}_{\mathrm{p}}$ in Relation zur maximal möglichen Entropie setzt $\left(\mathrm{H}_{\max }=\mathrm{ld}\left(\mathrm{N}_{\mathrm{m}}\right)\right.$; entspricht der Situation, wenn alle $\mathrm{N}_{\mathrm{m}}$ Materialien in gleicher Konzentration im Produkt vorkommen und keine Zerlegung möglich ist). Gemäß Gl. 4 kann auch die RPR der einzelnen Produktelemente $\left(\mathrm{RPR}_{\mathrm{j}}\right)$ errechnet werden, wobei dafür $\mathrm{H}_{\mathrm{p}}$ durch $\mathrm{H}_{\mathrm{j}}$ ersetzt werden muss.

Um fundierte Vergleiche zwischen unterschiedlichen Produkten zu ermöglichen, empfehlen Roithner et al. (2021) einen typischen produktspezifischen Materialienkatalog mit $\mathrm{N}_{\mathrm{m}}$ Materialien festzulegen, der dann $\mathrm{H}_{\max }$ für den Vergleich definiert (siehe Gl. 4).

$$
\begin{aligned}
& H_{j}=-\sum_{i=1}^{N_{m}} c_{i, j} \operatorname{ld}\left(c_{i, j}\right) \\
& H_{p}=\frac{1}{M_{p}} \sum_{j=1}^{N_{e}} M_{j} H_{j} \\
& R P R=1-\frac{H_{p}}{H_{\max }}=1-\frac{H_{p}}{\operatorname{ld}\left(N_{m}\right)}
\end{aligned}
$$

Ein RPR Ergebnis von 1 (= $100 \%)$ spiegelt eine maximale Recyclingfähigkeit bezogen auf das Produktdesign wider. Das Ziel ist somit, eine höchstmögliche RPR zu erreichen. Die RPR steigt, je stärker konzentriert einzelne 


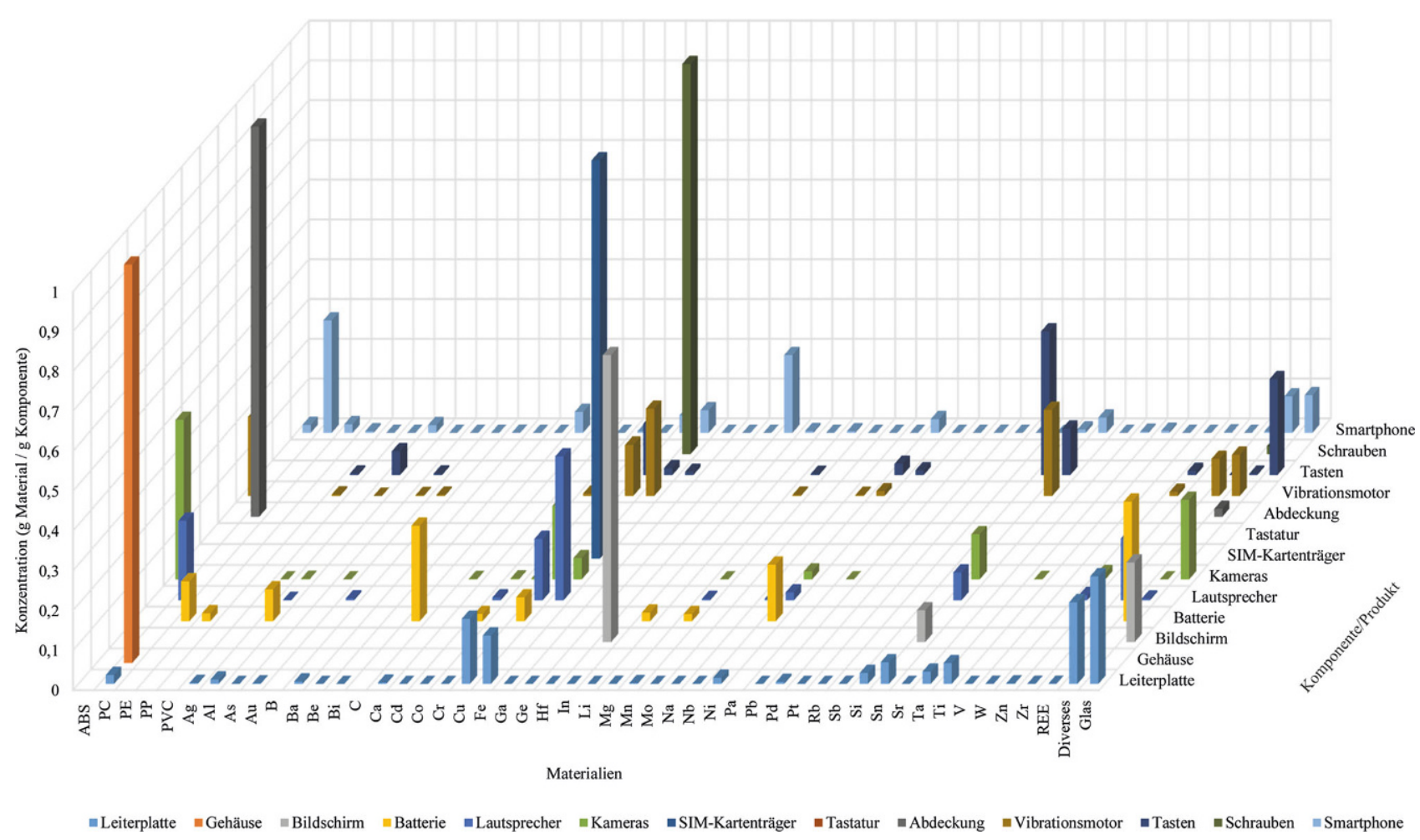

Abb. 3 Materialkonzentrationen in den Komponenten des Smartphones

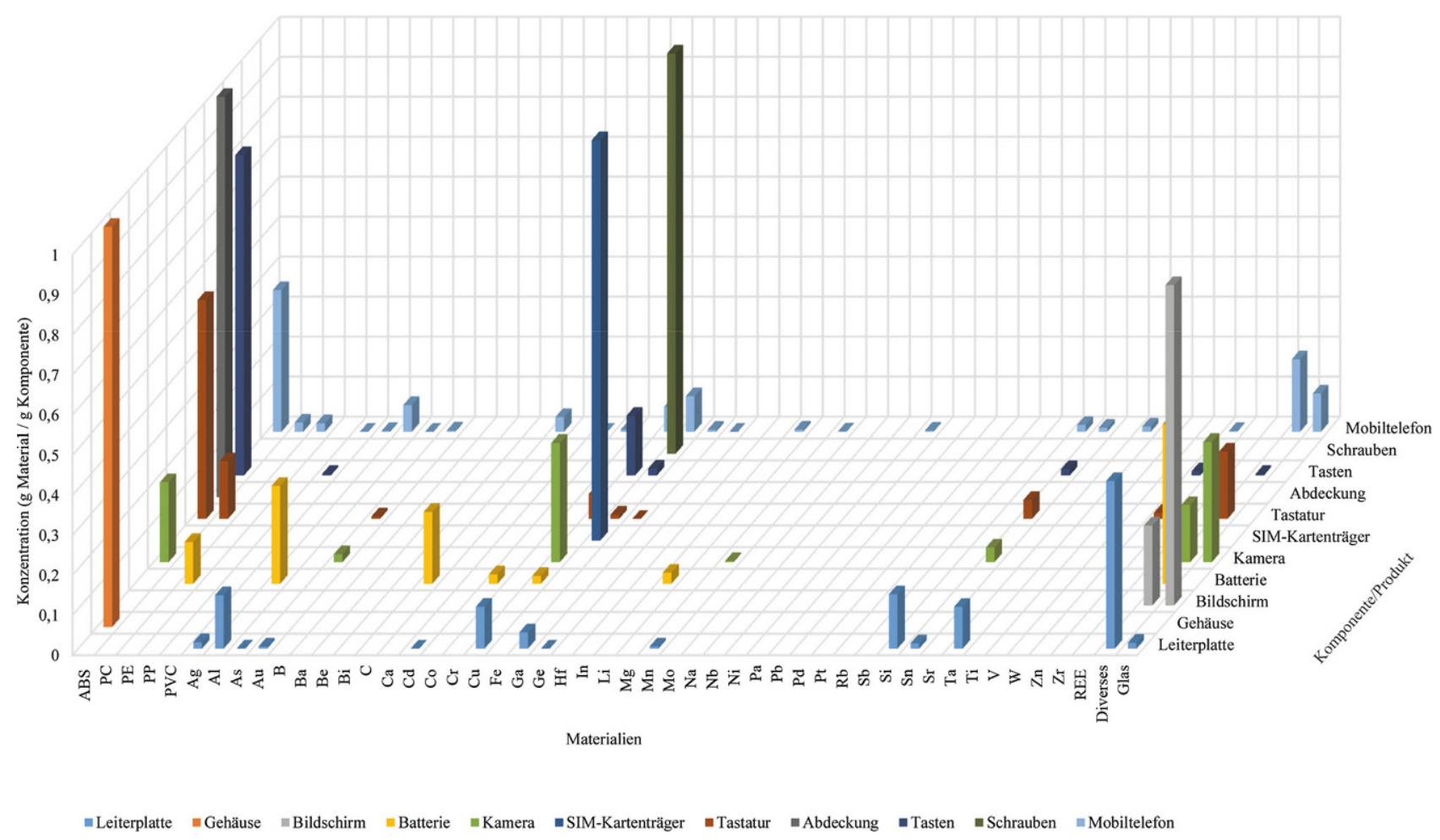

Abb. 4 Materialkonzentrationen in den Komponenten des Mobiltelefons 
Tab. 2 Relative produktinhärente Recyclingfähigkeit (RPR) der Mobiltelefone und der zugehörigen Komponenten (RPRi), sowie massegewichtete Beiträge der einzelnen RPR $R_{\mathrm{j}}$ zur RPR bei angenommener Zerlegung

\begin{tabular}{|c|c|c|c|c|c|c|c|}
\hline & $\begin{array}{l}\text { RPRj } \\
\text { (\%abs) }\end{array}$ & $\begin{array}{l}\text { RPRj } \\
\text { Beitrag } \\
\text { (\%abs) }\end{array}$ & $\begin{array}{l}\text { RPRij Beitrag } \\
\text { (\%rel) }\end{array}$ & & $\begin{array}{l}\mathrm{RPR}_{\mathrm{j}} \\
\text { (\%abs) }\end{array}$ & $\begin{array}{l}\text { RPRj } \\
\text { Beitrag } \\
\text { (\%abs) }\end{array}$ & $\begin{array}{l}\text { RPRij Beitrag } \\
\text { (\%rel) }\end{array}$ \\
\hline $\begin{array}{l}\text { Leiterplatte mit elektroni- } \\
\text { schen Bauteilen }\end{array}$ & 74,0 & 7,6 & 8,8 & $\begin{array}{l}\text { Leiterplatte mit elektroni- } \\
\text { schen Bauteilen }\end{array}$ & 86,5 & 14,7 & 16,1 \\
\hline Gehäuse & 100 & 17,0 & 19,5 & Gehäuse & 100 & 9,7 & 10,6 \\
\hline Bildschirm & 80,5 & 21,9 & 25,1 & Bildschirm & 87,1 & 7,0 & 7,7 \\
\hline Batterie & 84,0 & 18,6 & 21,2 & Batterie & 89,0 & 18,2 & 19,9 \\
\hline Kameras & 90,1 & 3,8 & 4,4 & Kamera & 87,2 & 8,4 & 9,2 \\
\hline SIM-Kartenträger & 100 & 1,7 & 1,9 & SIM-Kartenträger & 100 & 3,2 & 3,5 \\
\hline Abdeckung & 100 & 11,0 & 12,7 & Abdeckungen & 100 & 16,1 & 17,6 \\
\hline Tasten & 84,5 & 1,4 & 1,6 & Tasten & 95,1 & 3,1 & 3,4 \\
\hline Schrauben & 100 & 1,7 & 1,9 & Schrauben & 100 & 3,2 & 3,5 \\
\hline Lautsprecher & 82,0 & 1,0 & 1,2 & - & - & - & - \\
\hline Vibrationsmotor & 87,0 & 1,5 & 1,7 & - & - & - & - \\
\hline- & - & - & - & Tastatur & 81,7 & 7,7 & 8,4 \\
\hline Smartphone & - & 87,3 & 100 & Mobiltelefon & - & 91,3 & 100 \\
\hline
\end{tabular}

Materialien in den Produktelementen vorliegen, was durch die Zerlegbarkeit des Produkts begünstigt wird. Ein RPR von 0 bedeutet nicht, dass man das Produkt nachfolgend nicht recyceln kann, stellt jedoch in Hinblick auf das Produktdesign die schlechteste Situation dar.

\section{Ergebnisse}

Der für diese Bewertung gewählte Materialienkatalog orientiert sich an den 49 betrachteten Materialien des Smartphones $\left(=\mathrm{N}_{\mathrm{m}}\right)$, wodurch die maximale SE für beide Mobiltelefone mit $H_{\max }=\operatorname{ld}(49)$ fixiert ist (vgl. Gl. 4). Die Abb. 3 und 4 zeigen die Verteilung der Materialien in den Komponenten des Smartphones und des Mobiltelefons. Je weniger hohe Konzentrationssäulen für eine Komponente vorhanden sind, desto geringer ist ihre SE bzw. desto höher ist ihre $\mathrm{RPR}_{\mathrm{j}}$ (vgl. Tab. 2). Die Materialanzahl selbst lässt nur bedingt erkennen, ob die SE einer Komponente hoch ist oder nicht. Wenn viele Materialien in sehr geringer Konzentration vorliegen (wie z. B. bei der Leiterplatte des Smartphones, vgl. Abb. 3), dann haben diese Materialien, im Vergleich zu den bestimmenden Materialien mit hoher Konzentration, wenig Einfluss auf die SE bzw. RPR. Der beste Fall $\left(\mathrm{H}=0\right.$ bzw. $\left.\mathrm{RPR}_{\mathrm{j}}=1\right)$ ergibt sich, wenn eine Komponente aus nur einem Material besteht (z. B. die Gehäuse beider Mobiltelefone). Der schlechteste Fall $\left(\mathrm{H}=\mathrm{H}_{\max }\right.$ bzw. $\left.\mathrm{RPR}_{\mathrm{j}}=0\right)$ ergäbe sich, wenn alle Materialien in einer Komponente mit gleicher Konzentration vorkämen, d.h. alle Säulen gleich hoch wären.

Abb. 5 zeigt, dass bei der maximal angenommenen Zerlegung (bis zur Ebene der Sub-Sub-Komponenten) beide Mobiltelefone eine relativ hohe RPR erzielen, wobei jene des älteren Mobiltelefons geringfügig höher ist $(91,3 \%>87.3 \%)$ und dieses daher recyclingfähiger als das Smartphone ist. Die Differenz zwischen den RPR-Werten kann auf die bessere Konzentrierung von einzelnen Materialien in den Produktelementen des Mobiltelefons zurückgeführt werden (vgl. Abb. 3 und 4). Bei einer Zerlegung bis zur Komponenten-Ebene würden das Smartphone und das Mobiltelefon zufälligerweise eine praktisch idente RPR von $74,1 \%$ erzielen, obwohl ihre Materialverteilungen sehr unterschiedlich sind. Wären die Mobiltelefone nicht zerlegbar, würden sich beide RPR-Ergebnisse deutlich verringern, nämlich auf 40,8\% beim Smartphone und $46,3 \%$ beim Mobiltelefon, was die Wichtigkeit des Produktdesigns bzw. der Zerlegbarkeit herausstreicht.

Die RPR-Ergebnisse der Mobiltelefone und deren Komponenten sind in Tab. 2 gelistet. Bei Betrachtung der $\mathrm{RPR}_{\mathrm{j}}$ der einzelnen Komponenten werden Unterschiede zwischen den Mobiltelefonen deutlicher (vgl. Spalte 2 und 6 in Tab. 2). So erzielt beispielsweise die Leiterplatte des Smartphones eine $R P R_{j}$ von $74,0 \%$, während jene des Mobiltelefons eine $\mathrm{RPR}_{\mathrm{j}}$ von $86,5 \%$ erzielt. Dieser Unterschied bei den Leiterplatten ist einerseits auf die technologiebedingt höhere Materialan- zahl beim Smartphone (Smartphone $42>$ Mobiltelefon 19) und andererseits auf die tendenziell höhere Konzentrierung einzelner Materialien in den Sub-Elementen des Mobiltelefons zurückzuführen. Ähnliche Effekte können auch für die Komponenten Bildschirm, Batterie und Tasten beobachtet werden $\left(\mathrm{RPR}_{\mathrm{j}}\right.$ Smartphone $<$ Mobiltelefon). In beiden Mobiltelefonen erzielen die Komponenten Gehäuse, SIM-Kartenträger, Abdeckung/en und Schrauben eine $100 \%$ ige $R P R_{j}$, da sie aus Reinmaterialien bestehen bzw. in Reinmaterialien zerlegt werden können. Das Design dieser Komponenten ist außerdem in beiden Mobiltelefonen sehr ähnlich, was darauf hindeutet, dass sich deren Funktion über die Zeit nicht wesentlich verändert hat.

Zusätzlich kann für beide Mobiltelefone der relative Beitrag der einzelnen $\mathrm{RPR}_{\mathrm{j}}$ zur Produkt-RPR betrachtet werden (vgl. Spalte 4 und 8 in Tab. 2). Hierfür werden die $\mathrm{RPR}_{\mathrm{j}}$ der einzelnen Komponenten massegewichtet $\left(m i t \mathrm{M}_{\mathrm{j}} / \mathrm{M}_{\mathrm{p}}\right.$ ) und im Verhältnis zu RPR dargestellt. Es zeigt sich, dass sich die relativen Beiträge von einzelnen Komponenten stark unterscheiden. Beim Smartphone liefern die Komponenten Bildschirm $(25,1 \%)$, Batterie $(21,2 \%)$ und Gehäuse $(19,5 \%)$ die größten Beiträge zur RPR, während es beim Mobiltelefon die Komponenten Batterie (19,9\%), Abdeckungen $(17,6 \%)$ und Leiterplatte $(16,1 \%)$ sind. Die Höhe der Beiträge ist, aufgrund der ähnlichen $\mathrm{RPR}_{\mathrm{j}}$ der Komponenten (zw. 74 und $100 \%$ ), dabei stark von der Massengewichtung abhängig, wodurch z.B. die schwere- 
re Leiterplatte des Mobiltelefons einen höheren Beitrag liefert als jene des Smartphones.

\section{Schlussfolgerungen}

Der Vergleich von Mobiltelefonen auf Basis der RPR-Ergebnisse zeigt, dass sich die Bewertungsmethode von Roithner et al. (2021) dazu eignet, komplexe und schwer vergleichbare Produkte zu bewerten und folglich potenzielle Unterschiede in der produktinhärenten Recyclingfähigkeit festzustellen. Mit dieser Methode kann die RPR für das gesamte Produkt sowie für einzelne Produktelemente errechnet werden. Die RPR steigt, je konzentrierter Materialien in den zerlegbaren Produktelementen vorliegen. Die Produktergebnisse der Mobiltelefone zeigen, dass bezogen auf den gewählten Materialienkatalog beide Produkte eine relativ hohe RPR aufweisen, mit geringen Vorteilen für das Mobiltelefon (Smartphone rund $87 \%$; Mobiltelefon rund $91 \%)$. Die niedrigere RPR des Smartphones kann durch die komplexere Materialzusammensetzung bedingt durch die technologische Weiterentwicklung erklärt werden. Es zeigte sich, dass die RPR-Werte deutlich geringer ausfallen, wenn die modellierten Mobiltelefone nicht in deren Sub-Sub-Komponenten zerlegt werden (können). Die Ergebnisse der $\mathrm{RPR}_{\mathrm{j}}$-Beiträge der einzelnen Komponenten zeigen, dass für die Optimierung des Produktdesigns eine unterschiedliche Priorisierung gesetzt werden müsste. So könnte die höchste relative Steigerung der RPR beim Smartphone durch Optimierung des Bildschirms und beim Mobiltelefon durch Optimierung der Batterie erzielt werden, da es sich bei beiden Produktelementen um das schwerste Element des jeweiligen Produkts handelt. Es muss darauf hingewiesen werden, dass die Materialgruppe „Diverses“ so klein wie möglich gehalten werden sollte, da diese sonst die Ergebnisse maßgeblich beeinflussen könnte. Im Falle der modellierten Mobiltelefone war diese notwendig, um fehlende Konzentrationsdaten auszugleichen.

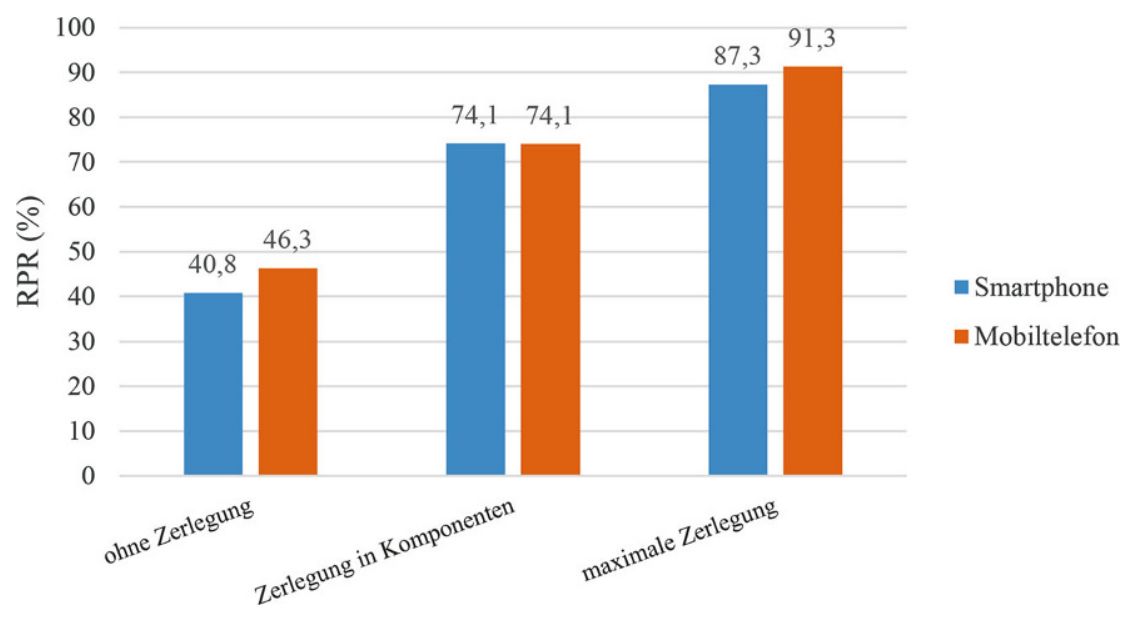

Abb. 5 Relative produktinhärente Recyclingfähigkeit (RPR) der Mobiltelefone nach den unterschiedlichen Zerlegungsschritten

ProduktdesignerInnen bzw. ProduzentInnen könnten durch Anwendung der RPR-Bewertungsmethode wesentliche Erkenntnisse über die produktinhärente Recyclingfähigkeit erlangen und unmittelbare Designoptimierungen planen bzw. durchführen (z.B. reduzierten und/oder konzentrierten Materialieneinsatz und hohe Zerlegbarkeit). Durch einen erhöhten Einsatz von trennbaren, hochkonzentrierten Materialien könnten so optimale Bedingungen für nachfolgende Verwertungswege geschaffen werden. Folglich könnten solche Produktoptimierungen $\mathrm{zu}$ einer gesteigerten Kreislaufführung von Produkten bzw. Materialien führen und zu einer effektiveren CE beitragen. Besonders für die Produktgruppe der Elektrogeräte wäre diese Entwicklung von Bedeutung, da in diesen Produkten diverse (wertvolle) Rohstoffe eingesetzt werden, welche derzeit nur teilweise rückgewonnen werden (können). Die von Roithner et al. (2021) angedachte Erweiterung der RPR-Bewertung um Eigenschaften wie Gefährlichkeit und Kritikalität von Materialen könnte für die Produktgruppe der Elektrogeräte relevante Erkenntnisse liefern, da bei dieser oftmals Schadstoffe (z. B. Flammschutzmittel) und kritische Rohstoffe (z.B. Kobalt) eingesetzt werden.
Funding Open access funding provided by TU Wien (TUW).

Open Access Dieser Artikel wird unter der Creative Commons Namensnennung 4.0 International Lizenz veröffentlicht, welche die Nutzung, Vervielfältigung, Bearbeitung, Verbreitung und Wiedergabe in jeglichem Medium und Format erlaubt, sofern Sie den/die ursprünglichen Autor(en) und die Quelle ordnungsgemäß nennen, einen Link zur Creative Commons Lizenz beifügen und angeben, ob Änderungen vorgenommen wurden.

Die in diesem Artikel enthaltenen Bilder und sonstiges Drittmaterial unterliegen ebenfalls der genannten Creative Commons Lizenz, sofern sich aus der Abbildungslegende nichts anderes ergibt. Sofern das betreffende Material nicht unter der genannten Creative Commons Lizenz steht und die betreffende Handlung nicht nach gesetzlichen Vorschriften erlaubt ist, ist für die oben aufgeführten Weiterverwendungen des Materials die Einwilligung des jeweiligen Rechteinhabers einzuholen.

Weitere Details zur Lizenz entnehmen Sie bitte der Lizenzinformation auf http://creativecommons.org/licenses/ by/4.0/deed.de. 
Bookhagen, B., Obermaier, W., Opper, C., et al (2018): Development of a versatile analytical protocol for the comprehensive determination of the elemental composition of smartphone compartments on the example of printed circuit boards. Anal Methods 10:3864-3871. https://doi. org/10.1039/c8ay01192c

Bookhagen, B., Bastian, D., Buchholz, P., et a (2020): Metallic resources in smartphones. Resour Policy 68:101750. https://doi.org/10.1016/j. resourpol.2020.101750

European Commission (2015a): Annex to the Communication From The Commission To The European Parliament, The Council, The European Economic And Social Committee And The Committee Of The Regions. Closing the loop-An EU action plan for the Circular Economy. Official Journal of the European Union. COM/2015/0614 final, 5 Seiten

European Commission (2015b): Communication From The Commission To The European Parliament, The Council, The European Economic And Social Committee And The Committee Of The Regions. Closing the loop-An EU action plan for the Circular Economy. Official Journal of the European Union. COM/2015/0614 final, 21 Seiten

European Union (2020): Circular Economy Action Plan: For a cleaner and more competitive Europe. Publications Office of the European Union, Luxembourg

Fontana, D., Pietrantonio, M., Pucciarmati, S. et al (2019): A comprehensive characterization of End-of-Life mobile phones for secondary material resources identification. Waste Manag 99:22-30. https://doi.org/10.1016/j.wasman. 2019.08.011

Holgersson, S., Steenari, B.M., Björkman, M. Cullbrand, K. (2018): Analysis of the metal content of small-size Waste Electric and Electronic Equipment (WEEE) printed circuit boards - part 1: Internet routers, mobile phones and smartphones. Resour Conserv Recycl 133:300-308. https://doi.org/10.1016/j.resconrec.2017.02.011 Laner ,D., Zoboli, O., Rechberger, H. (2017) Statistical entropy analysis to evaluate resource efficiency: Phosphorus use in Austria. Ecol Indic
83:232-242. https://doi.org/10.1016/j.ecolind. 2017.07.060

Liu, W., Ford, P., Uvegi, H., et al (2019): Economics of materials in mobile phone preprocessing, focus on non-printed circuit board materials. Waste Manag 87:78-85. https://doi. org/10.1016/j.wasman.2019.01.044

Palmieri, R., Bonifazi, G., Serranti, S. (2014): Recycling-oriented characterization of plastic frames and printed circuit boards from mobi-

le phones by electronic and chemical imaging. Waste Manag 34:2120-2130. https://doi.org/10. 1016/j.wasman.2014.06.003

Parchomenko, A., Nelen, D., Gillabel, J., et al (2020): Evaluation of the resource effectiveness of circular economy strategies through multilevel Statistical Entropy Analysis. Resour Conserv Recycl 161:104925. https://doi.org/10.1016/j. resconrec.2020.104925

Rechberger, H. (1999): Entwicklung einer Methode zur Bewertung von Stoffbilanzen in de Abfallwirtschaft. Vienna University of Technology

Rechberger, H., Brunner, P.H. (2002): A New, Entropy Based Method To Support Waste and Resource Management Decisions. Environ Sci Technol 36:809-16. https://doi.org/10.1021/ Technol

Rechberger, H., Graedel, T.E. (2002): The contemporary European copper cycle: statistical entropy analysis. Ecol Econ 42:59-72. https:// doi.org/10.1016/S0921-8009(02)00102-7 Roithner, C., Rechberger, H. (2020): Implementing the dimension of quality into the conventional quantitative definition of recycling rates. Waste Manag 105:586-593. https://doi.org/10. 1016/j.wasman.2020.02.034

Roithner, C., Cencic, O., Rechberger, H. (2021): Product design and Recyclability: How statistical entropy can form a bridge between these concepts. Veröffentlichung in Vorbereitung. Shannon, C.E. (1948): A Mathematical Theory of Communication. Bell Syst Tech J 27:623-656. https://doi.org/10.1002/j.1538-7305.1948.tb00917.

Singh, N., Duan, H., Yin, F., et al (2018): Characterizing the Materials Composition and Recovery
Potential from Waste Mobile Phones: A Comparative Evaluation of Cellular and Smart Phones. ACS Sustain Chem Eng 6:13016-13024. https:// doi.org/10.1021/acssuschemeng.8b02516 Smodiš, M., Samec, N., Kosec, B., et al (2018): The content of rare-earth elements in mobilephone components. Mater Tehnol 52:259-268. https://doi.org/10.17222/mit.2017.064

Sobańtka, A.P., Thaler, S., Zessner, M., Rechberger, H. (2014): Extended statistical entropy analysis for the evaluation of nitrogen budgets in Austria. Int J Environ Sci Technol 11:1947-1958. https://doi.org/10.1007/s13762-013-0401-2 Tan, Q., Dong, Q., Liu, L., et al (2017): Potential recycling availability and capacity assessmen on typical metals in waste mobile phones: A current research study in China. J Clean Prod 148:509-517. https://doi.org/10.1016/j.jclepro. 2017.02.036

Tanzer, J., Rechberger, H. (2020): Complex system, simple indicators: Evaluation of circularity and statistical entropy as indicators of sustainability in Austrian nutrient management. Resour Conserv Recycl 162:104961. https://doi.org/10. 1016/j.resconrec.2020.104961

Tarantili, P.A., Mitsakaki, A.N., Petoussi, M.A. (2010): Processing and properties of engineering plastics recycled from waste electrical and electronic equipment (WEEE). Polym Degrad Stab 95:405-410. https://doi.org/10.1016/j. polymdegradstab.2009.11.029

Ueberschaar, M., Dariusch Jalalpoor, D., Korf, N., Rotter, V.S. (2017): Potentials and Barriers for Tantalum Recovery from Waste Electric and Electronic Equipment. J Ind Ecol 21:700-714. https://doi.org/10.1111/jiec.12577

Hinweis des Verlags Der Verlag bleibt in Hinblick auf geografische Zuordnungen und Gebietsbezeichnungen in veröffentlichten Karten und Institutsadressen neutral. 